\section{Sistematización de la experiencia del Foro de Jóvenes por una Latinoamérica integrada, un espacio de diálogo intercultural en la ciudad de Rosario}

Dalila Emilse Capeletti

Universidad Nacional de Rosario, Argentina

dalila.capeletti@unr.edu.ar
Cultura(s) en clave de extensión universitaria / Intervenciones
RECEPCIÓN: 02/04/19

ACEPTACIÓN FINAL: 30/05/19

\section{Resumen}

Este artículo presenta una sistematización de la experiencia del Foro de Jóvenes por una Latinoamérica integrada desarrollado entre los años 2012 y 2016 y llevada adelante por la Universidad Nacional de Rosario. El mismo trata de una experiencia extensionista en integración sociocultural con las comunidades de inmigrantes latinoamericanos residentes en la ciudad. Con este objetivo, en primer lugar, daremos cuenta de las condiciones de contexto y las situaciones particulares que posibilitaron el desarrollo de la práctica. Luego, describiremos las acciones llevadas adelante con las comunidades de inmigrantes latinoamericanos residentes en Rosario y, finalmente, abordaremos los aprendizajes adquiridos durante la experiencia por los estudiantes involucrados en la práctica. A modo de cierre, reflexionaremos críticamente sobre las posibilidades de pensar y accionar una nueva práctica transformadora.

Palabras clave: relaciones internacionales, integración latinoamericana, extensión, sistematización de experiencias, perspectiva decolonial.
Systematization of experience of the Youth Forum for an integrated Latin America, a space for intercultural dialogue in the Rosario's city

\section{Abstract}

This article presents a systematization of experience of the Youth Forum for an Integrated Latin America developed between 2012 and 2016 belonging to the National University of Rosario. It is about an extension experience in social and cultural integration with Latin American immigrant communities living in the city. To this end, first of all, we will give an account of the context conditions and the particular situations that enabled the development of the practice. Next, we will describe the actions taken with the Latin American immigrant communities living in Rosario, and finally, we will address the learning acquired during the experience of the students involved in the practice. By way of closure, we will reflect critically on the possibilities of thinking and acting a new transformative practice.

Keywords: relation international ship, Latin America's integration, extension, systematization of experience, decolonial perspective.

\author{
Sistematização do Fórum de Jovens \\ por uma América Latina integrada, um \\ espaço de diálogo intercultural na cidade \\ de Rosario
}

\section{Resumo}

Este artigo apresenta uma sistematização do Fórum de Jovens por uma América Latina integrada desenvolvida nos anos de 2012 e 2016 na Universidade Nacional de Rosario. Trata-se de uma experiência de extensão em integração social e cultural com as comunidades de imigrantes latino-americanos que moram na cidade de Rosario. Com este objetivo, em primeiro lugar, daremos conta das condições de contexto e das situações particulares que possibilitaram o desenvolvimento da prática. Depois descreveremos as ações realizadas com as comunidades de imigrantes latino-americanos que moram em Rosario e finalmente, abordaremos as aprendizagens adquiridas na experiência pelos estudantes envolvidos na prática. Como encerramento, refletiremos criticamente acerca das possibilidades de pensar e agir em uma nova prática transformadora.

Palavras-chave: relações internacionais, integração latino-americana, extensão, sistematização de experiências, perspectiva decolonial.

Para citación de este artículo: Capeletti, D. (2019). Sistematización de la experiencia del Foro de Jóvenes por una Latinoamérica integrada, un espacio de diálogo intercultural en la ciudad de Rosario.+E: Revista de Extensión Universitaria, 9(10), 74-95. doi: 10.14409/extension.v9i10.Ene-Jun.8290. 


\section{Introducción ${ }^{1}$}

El Foro de Jóvenes por una Latinoamérica Integrada comenzó formalmente como un proyecto de extensión universitaria. El mismo tuvo dos ediciones y en ambas oportunidades se atravesaron instancias evaluativas, escritas y presenciales que resultaron aprobadas, por lo que contaron con el aval y la financiación de la Secretaría de Extensión Universitaria de la Universidad Nacional de Rosario (UNR). ${ }^{2}$

En este trabajo vamos a abordar al Foro como una experiencia extensionista de integración social y cultural latinoamericana. Hablar de experiencia nos permite abarcar aquellas relaciones y resultados alcanzados más allá de las actividades formalmente redactadas (formularios de presentación, evaluación y rendición técnica y financiera) en el marco de las Convocatorias. De esta manera, nuestro caso de estudio está conformado por todas aquellas acciones que han promovido y fortalecido la integración sociocultural latinoamericana realizadas por el Foro de Jóvenes por una Latinoamérica integrada entre noviembre de 2012 —momento de aprobación del proyecto- hasta septiembre de 2016, cuando se efectuaron las últimas actividades.

En primer lugar, presentaremos la perspectiva de análisis que guió el proceso de sistematización de la experiencia. Luego, daremos cuenta de las condiciones del contexto económico, social y político en el ámbito local, regional, nacional y mundial, como también de las situaciones particulares institucionales y organizacionales que posibilitaron el desarrollo de la práctica extensionista. A continuación, describiremos brevemente las acciones llevadas adelante con el objeto de favorecer la integración sociocultural con las comunidades de inmigrantes latinoamericanos residentes en Rosario y más adelante abordaremos los aprendizajes adquiridos durante la experiencia por los estudiantes involucrados en la práctica a partir del análisis de las entrevistas realizadas. Por último, reflexionaremos críticamente sobre las posibilidades de pensar y accionar una nueva práctica transformadora para la realidad de las comunidades de inmigrantes en la ciudad de Rosario.

\section{La ventana de posibilidades del trabajo con inmigrantes latinoamericanos en la ciudad de Rosario}

La riqueza de los encuentros que se sucedieron con las comunidades de inmigrantes latinoamericanos en el marco de las actividades del Foro de Jóvenes no fue lo suficientemente abordada desde la reflexión por el equipo coordinador durante la ejecución del proyecto. En ese momento no se tenía conocimiento de la herramienta de sistematización de experiencias, lo que impactó en la continuidad del Foro. "Sentíamos que teníamos que hacer algo más si queríamos continuar y teníamos que ponerle más energía. Faltó replantearse cosas con la participación de las comunidades" (Entrevistado 5, comunicación personal, 18 de septiembre de 2018).

1) Este artículo está basado en los resultados obtenidos en mi tesina de grado presentada para la obtención del título de licenciada en Relaciones Internacionales en la Universidad Nacional de Rosario, defendida en noviembre de 2018. 2) El primer proyecto se tituló "Foro de Jóvenes por una Latinoamericana Integrada" y fue presentado en la Convocatoria 2012, aprobado por Resolución Consejo Superior N 1088/2012. El segundo se llamó "Foro de Jóvenes por una Latinoamérica Integrada: integrando-nos con las comunidades en Rosario", Resolución Consejo Superior No 948/2014, Convocatoria 2014. 
Sin embargo, no deja de ser "un gran antecedente que se puede retomar" (ídem). Por todo esto, si queremos pensar nuevas acciones con las comunidades de inmigrantes latinoamericanos desde la disciplina de las relaciones internacionales, debemos partir obligatoriamente de la reflexión sobre la práctica en el marco de estos proyectos de extensión. Fue este convencimiento el que motorizó la investigación del caso.

Como afirman los directores de la propuesta:

"Proponer proyectos de extensión desde el campo disciplinar de las Relaciones Internacionales no es tarea sencilla, básicamente porque el pensamiento dominante tiende a alejar de los ambientes cotidianos la aplicación del análisis internacional, así como a estimular un criterio macro de análisis en el que el actor genuino y casi exclusivo —-todavía el único para muchos- es el Estado nacional". (Saavedra y Cianciardo, 2016:134)

Podemos observar que en el área de la integración regional (temática que se eligió para trabajar en el Foro) en sus dimensiones económica y política, esta situación cambió. Actores no gubernamentales, empresas transnacionales, grupos de presión, etc., fueron incorporados en los numerosos estudios que existen sobre los procesos integracionistas. Sin embargo, los aspectos social y cultural continúan siendo dimensiones pobres y no abordadas en profundidad por los internacionalistas.

$Y$ al escaso recorrido académico sobre estas dimensiones se suma otro problema. Lo que ocurre realmente es que, desde las relaciones internacionales, carecemos de un conocimiento que nos permita dar cuenta de la diversidad de actores involucrados en los procesos sociales y culturales de integración. En el caso que estudiamos aquí, diversos actores han aparecido a lo largo de la experiencia: organizaciones y movimientos sociales, comunidades de inmigrantes, centros y asociaciones culturales, empresas de traducción, en el caso brasileño con el portugués, los colegios de profesionales y estudiantes no organizados.

Desde la perspectiva analítica que adoptamos en este trabajo, entendemos que esta limitación se debe a que seguimos reproduciendo la idea de que la integración regional, en cuanto fenómeno histórico, es opuesta al conflicto. Para Quijano (2000), es posible que se den algunos procesos de subjetivación y formación de identidades no pacíficos sin que ello implique necesariamente el planteamiento de un conflicto frente al poder existente. Según la academia - podemos verlo expuesto en los programas del plan de estudio-, pareciera que uno empieza donde acaba el otro.

Esta concepción basada en un diagnóstico reducido de la realidad es el mecanismo que opera colonialmente sobre la integración latinoamericana en la disciplina de las relaciones internacionales. Se le adjudican diferentes categorías como ideal o utópica, nociones que dan cuenta de que la integración es planteada discursivamente como un fin que no podemos alcanzar, y en el fondo lo que se pretende es justificar "los medios" no integracionistas. Esta situación lleva a que conceptos como cooperación internacional tengan más llegada en la academia y la sociedad en su conjunto. En este sentido, es posible pensar la experiencia del Foro como una pequeña grieta que se abre en la producción de conocimiento disciplinar que coloca a los procesos integracionistas sociales y culturales en el centro del debate.

Uno de los primeros aportes teóricos de la práctica a la disciplina de las relaciones internacionales consistió en un nuevo concepto de integración regional construido colectivamente en el transcurso de los talleres. El Foro de Jóvenes por una Latinoamérica integrada 
entiende que: la integración regional debe ser un medio y no un fin en sí misma, que respete la diversidad latinoamericana y fortalezca las identidades tanto en sus modos de producción como en sus modos de consumo para que las personas sientan la necesidad de apropiarse del proyecto integrador. ${ }^{3}$

Desde esta perspectiva, podemos afirmar que el Foro se trató de un ejemplo de integración regional cuya particularidad es que contó con la presencia de numerosas situaciones conflictivas. Las mismas respondían a la lógica de los espacios interculturales en los cuales la diferencia (cultural) es su característica constitutiva. Por lo tanto, no se trataba de "crear" similitudes inexistentes, sino que se buscaba que lo común emergiera de un diálogo que resaltara lo distintivo de cada comunidad. Cuando esto se lograba, lo conflictivo se daba no entre las comunidades sino dentro de las colectividades mismas, llegando a interpelar la idea de Estado-nación.

Lo primero que debemos entender es que las diferencias culturales de nuestros pueblos son su componente cualitativo y no la justificación de sus desigualdades sociales. Es por esto que en el Foro las culturas latinoamericanas no eran consideradas solamente como distintas formas de mostrarse al mundo a partir de una vestimenta específica, una lengua, comidas, entretenimientos, trabajos, etc., posibles de ser patrimonializadas por la modernidad. Por el contrario, se afirma que esta concepción —muy presente en las instituciones estatales- (re)produce la colonización de los pueblos basándose en la idea de raza ${ }^{4}$ arraigada en el patrón de poder colonial donde la diferencia cultural justifica una jerarquía social.

La pregunta que surge desde el campo disciplinar de las relaciones internacionales es: ¿cómo podemos teóricamente hacer convivir en un mismo proceso de construcción colectiva conflicto e integración? Desde una visión decolonial, la propuesta sería pensarlos como las dos caras de una misma moneda, lo cual vence la idea del proceso como algo lineal y expresa la inevitabilidad de su existencia dependiente. Si no asumimos esto en la teoría seguiremos reproduciendo la binaridad colonial en la práctica, caracterizada en la disciplina de las relaciones internacionales por esquemas integracionistas formados por actores estatales con una predominancia de los ámbitos económicos y políticos.

La cultura occidental se estructuró y legitimó en torno al conocimiento científico moderno, pero este es solo uno de los tantos tipos de saberes presentes en nuestras sociedades. Las culturas son diferentes formas de concebir el mundo, de conocerlo, pensarlo, imaginarlo, por lo tanto no son objetos en el proceso de integración sino sujetos del mismo. Pensar en procesos integracionistas latinoamericanos desde una perspectiva decolonial implica que sea un proyecto visibilizador e inclusivo de esas diferencias y no homogeneizador de las mismas.

3) Este concepto fue un producto participativo del Primer Taller de Capacitación realizado en la ciudad de Rosario con la presencia de estudiantes de las doce Facultades de la Universidad Nacional de Rosario. En el Segundo Taller realizado el 16 de octubre de 2013, el concepto fue revisado y se reemplazaron dos términos modos de producción y modos de consumo. Fue discutido ampliamente y nos parece interesante mencionarlo puesto que creemos que ejemplifica un tema discursivamente importante para visibilizar la colonialidad en la disciplina. Los participantes del Foro entendían que producción y consumo eran dos términos rápidamente asociados a la economía capitalista y por lo tanto excluía otros modos. Por ello realizaron una redacción más explicativa definiéndolos como los distintos modos de percibir y satisfacer las necesidades básicas según el grupo social entendiendo que de esta manera era más inclusivo con la diversidad de identidades culturales presentes en la región.

4) El término de raza es nodal en el cuerpo de argumentación de la perspectiva decolonial. Para profundizar en el concepto y las implicancias del mismo en la decolonialidad latinoamericana, sugerimos la lectura del Capítulo 5 . La idea de raza. (Restrepo y Rojas, 2010) 
Como nos advierte Lander (2000), "la búsqueda de alternativas a la conformación profundamente excluyente y desigual del mundo moderno exige un esfuerzo de deconstrucción del carácter universal y natural de la sociedad capitalista-liberal" (p. 12). En esta dirección, una integración regional que sea plural, efectiva y participativa, empieza por buscar otras formas de construir la historia "perfiladas a recobrar las voces, experiencias y saberes formativos de los sujetos marginalizados por los enfoques dominantes que estructuran y validan una historia objetivista y eurocéntrica" (Díaz, 2010:225). Cabe destacar que "la crítica al eurocentrismo pasa por reconocer que todo conocimiento es un conocimiento situado histórica, corporal y geopolíticamente" (Galiardi, 2015:60). ${ }^{5}$

Una perspectiva crítica de la historia supone recuperar las voces, experiencias, saberes e ideales propios de los que habitaron el suelo latinoamericano y abrir así la posibilidad de reconocer otras formas de verla, comprenderla y configurarla. Da Silva y Orso (2010), en un trabajo titulado La evolución de la integración latinoamericana. Tres coyunturas históricas, 1810-1910-2010, plantean una lectura de las perspectivas tradicionales a partir del análisis de la historia de las ideas presentes en América Latina desde los procesos de independencia hasta la celebración de sus bicentenarios. En este estudio se sostiene que la idea de integración en la región latinoamericana surge en el contexto de las luchas independentistas y se encuentra imbuida por este espíritu. Entre los principales precursores se menciona a Francisco de Miranda (de Venezuela), Manuel Torres (neogranadino), Juan Martínez de Rozas (de Chile), y Simón Bolívar. ${ }^{6}$

Finalmente, debemos mencionar que la visión decolonial refiere a una ética y una política de la pluriversalidad, en oposición a diseños globales y totalitarios en nombre de la universalidad. La pluriversalidad constituye una apuesta por visibilizar y hacer viables la multiplicidad de conocimientos, formas de ser y de aspiraciones sobre el mundo (Galiardi, 2015:60). Por esta razón, la propuesta de integración regional del Foro requirió de un cuestionamiento de las pretensiones de objetividad y neutralidad de los principales instrumentos de naturalización y legitimación de la cultura occidental. El mecanismo desestabilizador utilizado fue el diálogo de saberes, ${ }^{7}$ que buscó descentrar el lugar ocupado por el conocimiento científico y disciplinar moderno en torno a la temática de la integración para pensar y resolver las pro-

5) En la temática de integración regional en América Latina, el abordaje que nos ofrece el campo subdisciplinar de la historia de las relaciones internacionales latinoamericanas puede resultar de gran utilidad para una comprensión crítica de la historia del fenómeno en la región (Da Silva et al, 2014). El objetivo es desestabilizar y debilitar la visión eurocéntrica que comporta la perspectiva de occidente que establece el comienzo de dicho proceso a mediados del siglo XX a partir de la materialización de procesos como Asociación Latinoamericana de Libre Comercio (ALALC), Pacto Andino y Asociación de Libre Comercio del Caribe (CARIFTA) cuyos antecedentes se registran en las Conferencias Panamericanas de 1889 a 1954. (Rosenthal, 1991; Mellado, 2002; Peña, 2011)

6) Este aporte es un importante antecedente para problematizar en futuras investigaciones desde una perspectiva decolonial, ya que se establece el inicio y por lo tanto las bases de los esquemas integracionistas en el preciso momento que comienza según Dussel (2000) la segunda Modernidad. Profundizar en el análisis de esta relación nos daría interesantes resultados para aportar a la historia de las relaciones internacionales latinoamericanas.

7) Al respecto, la extensión crítica propone el concepto de ecología de saberes propuesto por Sousa Santos (2010) a partir del cual se afirma que la diversidad social y cultural supone una diversidad epistemológica. "No significa renegar de la pertinencia del conocimiento científico o académico, sino incluir a otros saberes en los procesos de construcción de conocimientos socialmente significativos. La dimensión dialógica es un elemento fundamental en los procesos de construcción de nuevos conocimientos que puedan dar respuesta a los nuevos desafíos del presente" (Medina y Tommasino, 2018:248). 
pias problemáticas latinoamericanas, atender las necesidades que generan desigualdades y aumentar los márgenes de autonomía a partir de la reflexión sobre la práctica en la ciudad de Rosario. Esto se promovió a partir de distintos espacios de reflexión y construcción colectiva, interdisciplinarios e interculturales.

\section{Sistematización de la experiencia extensionista en integración regional sociocultural: el Foro de Jóvenes por una Latinoamérica integrada}

En primer lugar, debemos aclarar la diferencia existente entre el concepto tradicional de "sistematización" y lo que nosotros entendemos por "sistematización de experiencias". Mientras el primero refiere a clasificar, catalogar, ordenar datos e informaciones, el segundo se asume como una actividad reflexiva que todos hacemos al recuperar organizadamente la práctica con el objetivo de interpretar críticamente las experiencias vividas para extraer sus aprendizajes y compartirlos (Medina y Tommasino, 2018:16). Esta distinción permite ver que la sistematización de experiencias requiere de un esfuerzo cualitativamente más complejo que el que implica solamente organizar o clasificar datos, es decir, sistematizar informaciones. Según Jara (2018), la sistematización de experiencias es:

"Un ejercicio intencionado que busca penetrar en la trama próximo compleja de la experiencia y recrear sus saberes con un ejercicio interpretativo de teorización y de apropiación consciente de lo vivido. Requiere un empeño de curiosidad epistemológica y supone rigor metódico para convertir el saber que proviene de la experiencia, a través de su problematización, en un saber crítico, en un conocimiento más profundo. Para lograrlo, debemos generar un distanciamiento de la experiencia, que permita trascender la pura reacción inmediata frente a lo que vivimos, vemos, sentimos y pensamos. Así objetivizamos nuestra experiencia y al hacerlo, vamos encontrando sus vínculos con otras prácticas sociales de las que ella forma parte. La sistematización de experiencias permite ligar la reflexión que emerge de lo que vivimos con otras aproximaciones teóricas, para poder la comprender, más allá de la pura descripción o inmediatez, lo que estamos viviendo". (p. 55)

Es preciso conscientizarnos de la necesidad de esta herramienta en las experiencias extensionistas que llevamos adelante. Como nos advierte el autor, con estos procesos nos empoderamos, reconocemos los cambios que pueden darse $y$, sobre todo, entendemos que somos capaces de producir esos cambios. Es un trabajo que alimenta la imaginación creadora y nos habilita a pensar nuestras iniciativas como un ejercicio de poder, fortaleciendo nuestra capacidad de propuesta. A su vez, reconocemos y reforzamos nuestra capacidad de gestión de proyectos y analizamos críticamente el rol desempeñado por los distintos actores, por lo que podemos mejorar nuestras articulaciones y alianzas. Se trata de un proceso que no puede darse en condiciones de neutralidad sino que "está íntimamente vinculado a las relaciones de poder en que se debate la experiencia, y en la medida que se trata de un esfuerzo de apropiación crítica, propositiva y transformadora, la sistematización aportará a la construcción de nuevas relaciones de poder" (Jara:64).

Es así que, partiendo de la experiencia vivida, elaboramos un plan de sistematización para la experiencia extensionista que teníamos desarrollada. Para ello, nos guiamos con las etapas propuestas en "Extensión crítica: construcción de una Universidad en contexto. 
Sistematización de experiencias de gestión y territorio de la Universidad Nacional de Rosario" (Medina y Tommasino, 2018), esquematizadas en el Cuadro 1.

En una primera instancia definimos el objeto, el objetivo y el eje de abordaje. Lo que elegimos recuperar fue la propuesta pedagógica del Foro de Jóvenes por una Latinoamérica integrada para el tratamiento de la integración latinoamericana llevada adelante con las comunidades de inmigrantes latinoamericanos residentes en la ciudad de Rosario desde noviembre de 2012 a septiembre de 2016. Luego, definimos el objetivo de sistematización, cual es contribuir a la reflexión teórica con conocimientos surgidos directamente de la

Cuadro 1. Etapas de la sistematización de experiencias.

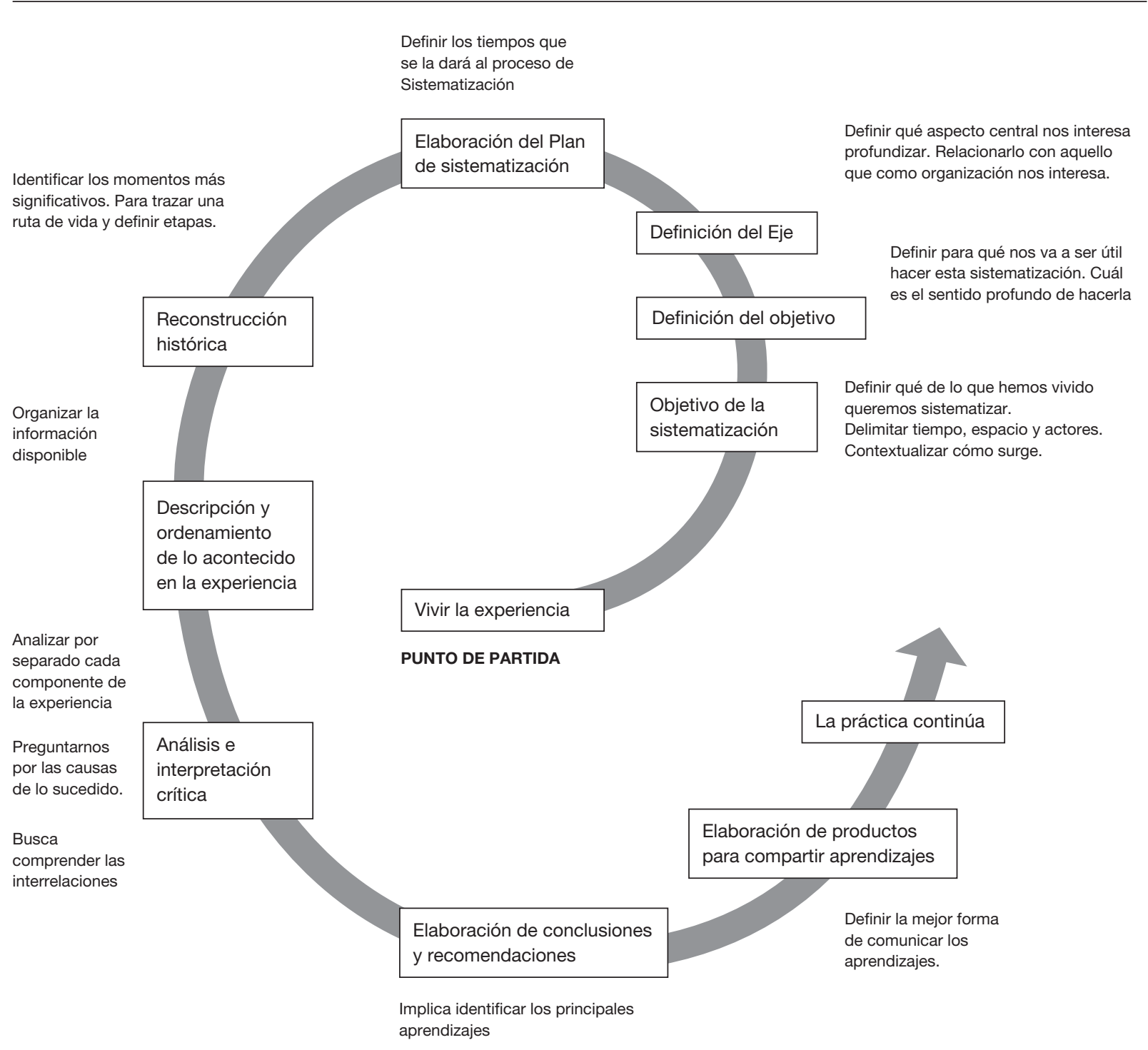

Fuente: Zúñiga López; Zúñiga Preciado (2013). Metodología para la Sistematización Participativa de Experiencias Sociales. Una propuesta desde la Educación Popular. IMDEC. 
experiencia. Y finalmente identificamos el eje, es decir, el aspecto central que queríamos profundizar, siendo nuestro interés reconocer cuáles son las potencialidades y los aprendizajes con relación a la formación profesional y la construcción de conocimiento en las relaciones internacionales en la UNR.

Superada esta primera etapa de planificación, nos sumergimos en la reconstrucción histórica del proceso extensionista, puesto que "la experiencia siempre está constituida por acciones; es decir, por cosas que hacemos (o dejamos de hacer) las personas. De forma intencionada o inintencionada; planificada o imprevista; dándonos cuenta de su realización o sin reconocerla mientras las realizamos" (Jara, 2018:53). Para ello, utilizamos la técnica metodológica de la matriz cronológica, la cual fue diseñada a partir de la lectura de los registros y las memorias de los talleres, las actas de las reuniones, los documentos de planificación, diseños metodológicos, fotografías y videos, publicaciones en redes sociales, notas periodísticas, artículos y ponencias. En este trabajo presentaremos una breve descripción de los datos allí recopilados a fin de facilitar la lectura y poder abocarnos con mayor profundidad al análisis de los mismos.

Los siguientes apartados consisten en el análisis e interpretación crítica de la experiencia a partir de la cual buscamos determinar los aspectos innovadores de la práctica de extensión en el abordaje de la integración sociocultural latinoamericana. Para ello fue necesario enriquecer los datos con información de los miembros del equipo que participaron activamente en las dos ediciones del Foro, "en toda experiencia están presentes las percepciones, sensaciones, emociones e interpretaciones de cada una de las personas que viven esas experiencias, es decir, de sus protagonistas" (p. 53). Así, realizamos entrevistas a cinco estudiantes avanzados en Relaciones Internacionales a fin de identificar los aspectos distintivos que tuvo esta experiencia en el ámbito de su formación profesional. Las mismas serán presentadas en el sexto apartado.

\section{Condiciones de posibilidad}

La sistematización de experiencias entendida como "procesos históricos y complejos en los que intervienen diferentes actores y que se llevan a cabo en un contexto económico, social y cultural determinado, y en situaciones organizativas o institucionales particulares" nos invita a identificar el contexto no como algo exterior a la experiencia sino como una de las dimensiones que acompañaron el desarrollo del Foro. "El momento histórico, el espacio geográfico y el entorno sociocultural son la condición de posibilidad de cada experiencia, fuera de los cuales no es factible entenderla, pues forman parte de su realización" (Jara, 2018:52).

En este apartado nos referiremos, primero, a las condiciones de contexto económico, social y político en los ámbitos local, regional, nacional y mundial, en los que se desenvolvió el Foro de Jóvenes por una Latinoamérica integrada, y luego mencionaremos algunas situaciones particulares (institucionales, organizacionales, grupales o personales), esto es, aquellas circunstancias en las cuales se vivió la experiencia y que le dieron su dimensión propia e irrepetible.

El escenario internacional está marcado por la crisis económica y financiera de 2008 originada en Estados Unidos cuyas consecuencias se extendieron a nivel global como a lo largo del tiempo. Según advierte Tokatlian (2011), lo relevante de los múltiples efectos de esta crisis es que expresan y dinamizan una redistribución del poder internacional caracterizado por: 1) 
el gradual e intensificado traslado del centro del poder de Occidente a Oriente, con China e India como nuevos actores dinámicos en la economía mundial, lo que orienta la geopolítica mundial cada vez más hacia Asia; 2) la tendencia a la multipolaridad se superpone a espacios estrictamente unipolares, como es el caso del ámbito militar donde nadie tiene todavía la capacidad de contrabalancear, en solitario o mediante una alianza cohesiva, a Estados Unidos; y 3 ) en términos ideológicos, hay un escenario bifurcado que puede conducir a un progresismo defensivo o a un avance reaccionario.

A nivel regional, Rouquié (2011) identifica tres cambios decisivos que han contribuido a crear un círculo virtuoso que configuró históricamente una "nueva América Latina" (p. 111). En primer lugar, la estabilidad política y la democracia, dado que nunca en su historia América Latina había tenido tantos gobiernos democráticos y durante tanto tiempo. Esto es seguido por los avances económicos y sociales que se vieron beneficiados entre 2003 y 2008 por una bonanza económica singular debido a la fuerte demanda asiática y el alza de precio de las commodities. Finalmente, existió una capacidad de resistencia a los choques externos, en especial al impacto de la crisis financiera internacional, con algunas breves excepciones, como en el caso de México.

A su vez, la región presentó un cambio ideológico que se conoció metafóricamente como "giro a la izquierda" (Sader, 2009). Si bien hubo países que siguieron optando por el modelo liberal (México, Colombia, Perú), en Sudamérica —y Nicaragua - fueron elegidos democráticamente gobiernos progresistas para enfrentar las consecuencias del modelo neoliberal hegemónico en la región. En 1999 Hugo Chávez asumió la presidencia en Venezuela y luego se sumaron Lula Da Silva en Brasil, Néstor Kirchner y Cristina Fernández en Argentina, Rafael Correa en Ecuador, Evo Morales en Bolivia, Fernando Lugo en Paraguay, Michelle Bachelet en Chile, y Tabaré Vázquez y José Mujica en Uruguay.

Como afirma Rouquié (2011), lo importante de este momento es que los gobiernos de la región comenzaron a sentirse más seguros de sí mismos y esto se vio reflejado cuando obstaculizaron la consagración del Área de Libre Comercio de las Américas (ALCA) propuesta por Washington en la Cumbre de las Américas de Mar del Plata en 2005, y también, a partir de la consolidación de esquemas de integración regional propios sin la presencia norteamericana -UNASUR, ${ }^{8}$ ALBA $^{9}$ y Celac- $-{ }^{10}$ protagonizando un bloque de fuerzas frente a los intereses de Estados Unidos en la región. Sumado a ello, en el escenario internacional los Estados de América Latina se han convertido en socios indispensables para legitimar las concertaciones multilaterales con objeto de mejorar la gobernanza mundial, y esto puede atestiguarse con la presencia de Argentina, Brasil y México dentro del G20.

Por su parte, el ámbito nacional no escapó al contexto regional, y en diciembre de 2011 Cristina Fernández asumió un segundo mandato fortalecido por el amplio apoyo popular, lo que la llevó a profundizar las relaciones con la región, principalmente con Brasil y Venezuela, desde una vocación marcadamente integracionista.

En el escenario local debemos tener presente que Rosario es una ciudad metropolitana, la tercera más grande del país, con un puerto internacional y una de las universidades públicas

8) Unión de Naciones Sudamericanas.

9) Alianza Bolivariana para los Pueblos de Nuestra América.

10) Comunidad de Estados Latinoamericanos y Caribeños. 
más prestigiosas de América Latina, lo que la coloca como un destino elegido por numerosos latinoamericanos ya sea para estudiar, trabajar, conocer o disfrutar.

Lo antedicho demuestra que las condiciones de contexto internacional, regional, nacional y local eran propicias para la puesta en práctica de una propuesta integracionista como la del Foro, y como veremos, también fue favorecida por las circunstancias particulares que acompañaron su origen.

La primera de ellas fue que el proyecto estuvo impulsado por estudiantes de Relaciones Internacionales de la UNR miembros de la organización nacional 13 Ranchos. ${ }^{11}$ Esta tuvo desde sus inicios una vocación integracionista y latinoamericana ya que se creó en 2001 en Catamarca, luego de la participación de lo que fueron sus fundadores en el Foro Social Mundial de Porto Alegre. Desde 2003 a 2007, la organización coordinó el Foro de Juventudes Políticas del Mercosur (FJPM), un espacio abierto de encuentro plural y político de las juventudes progresistas del Mercosur contra el neoliberalismo y el dominio del mundo por el capital o por cualquier forma de imperialismo. En la Declaración de Asunción (2003), el FJPM proclamó como su objetivo:

"Consolidar una regionalización solidaria, que — como una nueva etapa en la historia de nuestras naciones - respete los derechos humanos de todos los ciudadanos y ciudadanas de nuestros pueblos y al medio ambiente, apoyándose en sistemas e instituciones internacionales democráticos que estén al servicio de la justicia social, de la igualdad y de la soberanía de los pueblos promoviendo la participación social en el proceso de integración regional". (s/p)

Además de la impronta ideológica que acompañó el proyecto, la 13 Ranchos, dada su trayectoria en educación popular, fue la organización contraparte que facilitó las herramientas metodológicas para la concreción de las actividades.

Por otra parte, la segunda situación particular que favoreció la propuesta fue que se oficializó como un proyecto de extensión universitaria. Esto, por un lado, fue innovador en la formación de profesionales en Relaciones Internacionales, puesto que ni el plan de estudios de la carrera ni los programas de las materias incluyen como una instancia acreditable la extensión. Por otro lado, darle un marco institucional en el ámbito educativo nos permitió alcanzar con mejores resultados la vinculación con los distintos actores involucrados en la temática integracionista -estudiantes latinoamericanos, organizaciones de inmigrantes, consulados, etcétera.

\section{Integrar-nos para conocer-nos}

La experiencia extensionista se formalizó a partir de dos proyectos de extensión universitaria en el marco de las Convocatorias "La Universidad y su compromiso con la sociedad" de la Secretaría de Extensión de la UNR. A continuación se presentan los principales lineamientos y una descripción de las diferentes actividades desarrolladas en cada propuesta de trabajo.

11) Es una organización social de alcance nacional y de convicción federal profundamente política, que concibe a ésta como la herramienta necesaria para la transformación social de nuestros pueblos. Las banderas que la 13 Ranchos sostiene a nivel nacional son: Democracia Participativa; Equidad de Género; Ambiente Sustentable; Inclusión Social e Integración Latinoamericana. Véase https://www.facebook.com/13Ranchos/ 


\section{a) Foro de Jóvenes por una Latinoamérica integrada (primera edición)}

El objetivo general de esta primera parte fue promover un espacio de reflexión en la comunidad académica para el tratamiento de la temática de integración regional con los estudiantes de las 12 facultades de la UNR. Para ello se propuso crear participativamente una agenda de temas de interés que procuraba evitar situarse: "en el lugar del saber autorizado en el tema, para habilitar en primer lugar un diálogo abierto, sincero y ubicado genuinamente en el territorio de la multi y también interdisciplina y a partir de allí, en segundo lugar, producir una síntesis superadora y construida colectivamente" (Saavedra y Cianciardo, 2016:39).

El resultado que se esperaba era la conformación de un equipo interdisciplinario que garantizara la continuidad del proyecto en una segunda etapa en la que se trabajaría directamente con las comunidades de inmigrantes. Este grupo contaría con estudiantes latinoamericanos que estuvieran estudiando en la UNR, los cuales serían el nexo con las diferentes colectividades.

Se planteó "una logística dual de ejecución que involucraba acciones internas de sensibilización y capacitación destinadas al equipo ejecutivo de estudiantes del proyecto; y acciones externas o públicas, destinadas a la comunidad estudiantil de grado de la UNR" (Saavedra y Cianciardo:137).

Las acciones internas consistieron en dos talleres que buscaron consensuar el qué y el cómo del objetivo del proyecto. Primero, se llevó adelante un encuentro cuya tarea fue indagar, recuperar y sistematizar la diversidad de nociones en torno al tema de la integración latinoamericana surgidas desde la propia diversidad disciplinaria, ${ }^{12}$ para luego construir participativamente un concepto consensuado con el que iba a trabajar el Foro. Este trabajo de producción teórica era necesario puesto que al iniciar el proyecto no se contaba, desde Relaciones Internacionales, con un término que pudiera dar cuenta de la complejidad de esta propuesta de integración regional que pensaba la misma como un proceso que surgía desde las bases de los sectores sociales y no a partir de políticas gubernamentales. El recorrido es inverso. Las dimensiones social y cultural de la integración alimentan, fortalecen y legitiman los ámbitos político y económico del proceso.

El otro encuentro estuvo relacionado con cómo se iba a llevar adelante esta propuesta a partir de la pedagogía de Paulo Freire y se acordó que, mediante los talleres sustentados en la metodología de educación popular, se viabilizaría una posibilidad de abordaje desde una construcción democrática del conocimiento, en la búsqueda de un fortalecimiento del ejercicio de la ciudadanía, incluyendo en este ejercicio la identidad/identificación como ciudadanos latinoamericanos (Baroni et al., 2014). Es importante destacar que en ambas instancias fue fundamental el diálogo con la organización contraparte, puesto que la metodología fue trabajada conjuntamente.

Una vez definidos colectivamente el qué y el cómo de Foro de Jóvenes por una Latinoamérica integrada se realizaron las acciones públicas o abiertas que requirieron consensuar

12) En el informe final presentado a la Secretaría de Extensión, los miembros del proyecto afirman que acordaron "en priorizar la diversidad de estudiantes y docentes participantes antes que la cantidad" habiendo logrado la participación sostenida de "estudiantes, docentes y extensionistas de carreras como Psicología; Ingenierías (Electrónica, Mecánica, Electricista y Civil); Arquitectura; Derecho; Antropología; Filosofía; Historia; Cs. De la Educación; Medicina, Enfermería; Odontología; Cs. Agrarias; Cs. Veterinarias; Licenciatura en Matemática; y por supuesto, Comunicación y Trabajo Social" (Saavedra y Cianciardo, 2016:136). 
el para qué y el con quién de la propuesta extensionista. Los destinatarios directos eran los estudiantes de la UNR y las comunidades de inmigrantes latinoamericanos que vivían en Rosario. A su vez, indirectamente afectaba a toda la comunidad académica y a la ciudad. Primero, se realizó un taller interfacultades que propuso detectar temas de agenda sobre la integración latinoamericana que interesaran a los estudiantes de cada disciplina a fin de determinar participativamente las cuestiones a abordar en el Foro. Una vez procesada la información por el equipo de ejecución, los ejes construidos fueron: a) Educación formal universitaria: repensar el lugar de la educación formal universitaria en la construcción de la integración latinoamericana. Asimismo, debatir si la misma excluye o no prácticas educativas no formales; b) Ambiente y desarrollo: reflexionar en torno a una propuesta de desarrollo integral y amigable con el planeta en Latinoamérica; c) Conectividad e infraestuctura: analizar los modos de interconexión y comunicación en el territorio latinoamericano y sus efectos en la integración de los pueblos; y d) Cultura e interculturalidad latinoamericana: debatir sobre la existencia de una identidad latinoamericana: ¿se puede hablar de una cultura Latinoamérica o de muchas culturas interrelacionadas?

En este Foro, cada eje temático fue identificado como una estación. Así, las estaciones permanecían fijas y estaban conformadas por un docente/investigador/especialista y un extensionista con el objetivo de poner en diálogo la teoría y la práctica en cada temática abordada. "Los que se movían eran los grupos de personas que debían pasar por todas ellas. De esta manera, los grupos de participantes debieron trabajar en la consecución del objetivo que debían cumplir todos diferentes migrando de estación en estación" (Saavedra y Cianciardo, 2016:140). A su vez, un facilitador miembro del equipo del Foro estaba encargado de garantizar en todo momento el diálogo horizontal e inclusivo de las diferentes miradas participantes.

Todos los asistentes fueron divididos en cuatro grupos a los cuales se les asignó una tarea específica a lo largo de la experiencia y, cuando se concluyó con la dinámica, se realizó una puesta en común acerca de los que se había concluido:

"Se trabajó identificando horizontes, montañas, lagunas y raíces de cada agenda definida en el proceso de ejecución del proyecto. La metáfora de discutir sobre los horizontes, tenía que ver con las propuestas, posibilidades y metas que los asistentes relacionaban entre la agenda en discusión y la integración regional; mientras que reflexionar sobre montañas arribaría a conclusiones en torno a obstáculos y desafíos del tema discutido con respecto a la integración latinoamericana. Por su parte, el diálogo sobre lagunas condujo a identificar vacíos y debilidades en el propósito integracionista, así como también la reflexión en torno a las raíces remitía a los logros visibilizados en el proceso de integración en América Latina". (Saavedra y Cianciardo:140)

En tanto, la experiencia también tuvo propuestas interculturales que buscaron promover un espacio de intercambio con las comunidades latinoamericanas en general, no solo con las que tenían un vínculo con la Universidad. Uno de ellos estuvo incluido en la agenda de actividades del proyecto y consistió en la realización de un Festival Latinoamericano que contó con la participación de dos grupos musicales latinoamericanos. Y otro fue propuesto a partir de los vínculos generados en el transcurso del proceso con el Ingeniero Agrónomo argentino 
Ricardo Zehnder, quien, en camino a Santa Cruz, Bolivia, para presenciar el G77+China, ${ }^{13}$ decidió realizar una mesa de diálogo para compartir las experiencias que ambos equipos estaban llevando adelante en su territorio. La misma congregó a miembros de la nación Tupí Nambá del nordeste brasileño, entre los cuales había líderes políticos, como el cacique Ramon Tupinambà; representantes de organizaciones sociales, como Joselita da Casa da Economia Solidaria; Grasiele, de la Tea Agroecológica, comprometidos con el desarrollo sustentable en América Latina y los miembros del equipo ejecutivo del Foro.

El resultado de esta primera edición del proyecto arrojó un saldo positivo. En primer lugar, se constituyó un equipo de trabajo como se había previsto, conformado por 25 miembros entre los cuales se encontraban estudiantes, docentes y graduados de las carreras de Relaciones Internacionales, Ciencia Política, Psicología, Derecho, Medicina, Ingeniería, Traductorado en Portugués y Kinesiología. A su vez, eran miembros de diferentes colectividades latinoamericanas: brasileña, chilena, colombiana, uruguaya y argentina (ver Figura 1). Algunos de los miembros estaban organizados o participaban de alguna asociación que representaba a su comunidad, aunque en su mayoría se trataba de inmigrantes independientes. Por otra parte, la organización contraparte salió fortalecida de esta primera experiencia extensionista. Esto quedó evidenciado en la participación que tuvo la organización nacional en el Foro interfacultades, donde se convocó a miembros de la 13 Ranchos de Córdoba, Catamarca y Buenos Aires.

Figura 1. Países participantes del Foro de Jóvenes por una Latinoamérica integrada.

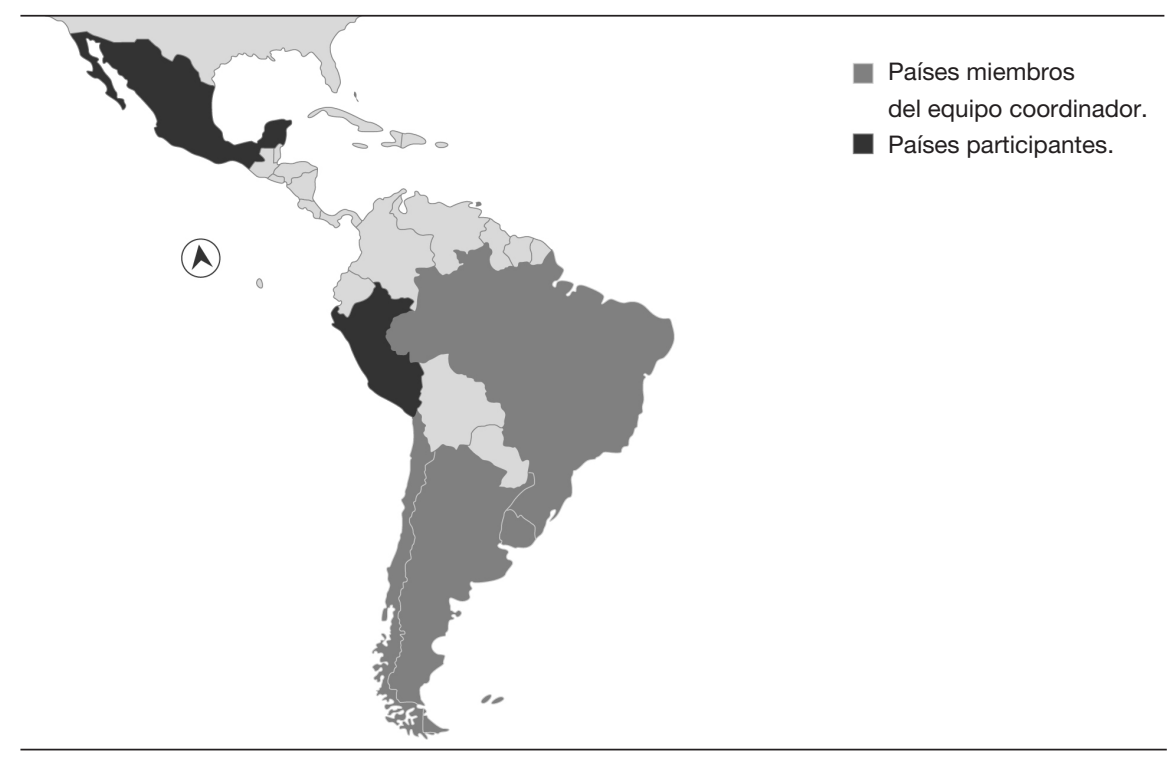

Fuente: elaboración propia.

13) Se trató de la Cumbre Extraordinaria de Jefas y Jefes de Estado y de Gobierno G77 + China, celebrada en Santa Cruz y con la asistencia de presidentes e importantes líderes políticos a nivel mundial. El Grupo de los 77 (G-77) es la mayor organización intergubernamental de países en desarrollo en las Naciones Unidas, que proporciona los medios para que los países del sur puedan articular y promover sus intereses económicos colectivos y mejorar su capacidad de negociación conjunta sobre los principales temas económicos internacionales en los Estados Unidos, sistema de las Naciones Unidas, y promover la cooperación sur-sur para el desarrollo. 
En otro orden de cosas, si bien existía desde el equipo conformado mucha voluntad y entusiasmo para continuar con el trabajo que se venía realizando, se reconocía que para ello se necesitaba profundizar, desde la teoría y la historia, de qué hablamos cuando nos referimos a extensión. De esta manera, se organizó un taller de formación en extensión universitaria destinado a los miembros del Foro de Jóvenes por una Latinoamérica integrada a cargo del equipo de gestión de la Secretaría de Extensión de la UNR.

En esta actividad se conversó sobre la participación del equipo del Foro en el VI Congreso Nacional de Extensión a realizarse en la ciudad de Rosario, el cual nuclearía a universitarios y miembros de organizaciones sociales de toda Latinoamérica. Fue así que surgió la propuesta de llevar adelante un Foro de Jóvenes Extensionistas Latinoamericanos y Caribeños con el objetivo de conocer el estado de la integración regional en la educación superior y compartir la situación de los estudiantes extensionistas en las diferentes universidades latinoamericanas. Dicho espacio, desarrollado en septiembre de 2014, marcó el primer antecedente de un encuentro extensionista exclusivo de estudiantes. Asimismo, otorgó una mayor presencia al Foro en los espacios de extensión nacional y latinoamericanos y contribuyó al fortalecimiento del vínculo institucional con la gestión de la Universidad.

\section{b) Foro de Jóvenes por una Latinoamérica integrada (segunda edición)}

El objetivo general de esta segunda edición del proyecto buscó consolidar los resultados de la primera y continuar aportando al fomento y difusión de la temática integracionista en la comunidad de Rosario. Se seleccionaron las cuatro comunidades de inmigrantes que tuvieron una participación continua en las actividades de la primera edición del proyecto con el objetivo de profundizar el vínculo construido a lo largo de esos meses. De esta manera, se fortaleció la relación con las comunidades chilena, brasileña, uruguaya y colombiana. Las actividades se pensaron y organizaron en conjunto con los actores de cada comunidad. Se propuso: a) generar espacios de diálogo basados en los ejes temáticos surgidos en el Foro 2014; b) elaborar propuestas de acción sobre la base de las problemáticas comunes surgidas en dichos espacios; y c) difundir las problemáticas comunes y las propuestas generadas en conjunto a la comunidad de Rosario.

Como resultado se planificaron cinco encuentros que funcionaron como espacios interculturales organizados conjuntamente con los actores de las comunidades de inmigrantes seleccionadas. El primero consistió en un taller vivencial donde se trabajó la apropiación del territorio — la ciudad de Rosario- a partir de la herramienta metodológica del sociodrama. ${ }^{14}$ La temática escogida por el equipo fue la noción de desarraigo entendida como una problemática común a todos los participantes del Foro, incluso a aquellos miembros argentinos pero que no habían nacido en la ciudad de Rosario.

El segundo encuentro se organizó y gestionó con la comunidad de inmigrantes chilenos y contó con la participación de los diferentes actores locales de la comunidad chilena: el cón-

14) Según Zuretti (2010), el sociodrama es una herramienta de trabajo grupal que permite comprender el proceso más allá de su estructura aparente, llegar a sus raíces más profundas y alcanzar el conocimiento escondido trayéndolo a la superficie. Se incluyen los procesos individuales como medio para lograr un cambio en la estructura total. "En sociodrama son los roles sociales los que serán explorados, pero no bajo la perspectiva de experiencias individuales o privadas, sino en modos que sean internalizadas como un grupo" (Morineau, 2010:180). 
sul Mg. Marcelo Aliaga, la Asociación cultural de residentes chilenos en Rosario, miembros de la comunidad Mapuche, profesores, estudiantes y trabajadores chilenos no organizados. El tercer encuentro unió a dos comunidades, la colombiana y la uruguaya. Y finalmente, una tercera instancia tuvo como protagonista a la comunidad de brasileños.

Cada uno de estos espacios tuvo un momento dedicado al trabajo reflexivo en formato de taller, en el cual la temática y las dinámicas fueron seleccionadas previamente por los miembros del equipo ejecutivo del foro y los participantes de la comunidad que protagonizaba el encuentro. El objetivo era fortalecer a cada comunidad latinoamericana promoviendo espacios de intercambio con otras comunidades donde expondrían sus culturas, costumbres, pensamientos y problemáticas concretas. En los mismos se alentaría el diálogo y la reflexión crítica con el fin de identificar puntos de cercanía con las otras comunidades como ciudadanos latinoamericanos. La finalidad de conocer y problematizar la situación que cada comunidad de inmigrantes tenía en la ciudad de Rosario era utilizada para incentivar la toma de decisiones y la realización de acciones concretas para transformar ese diagnóstico de la realidad.

Un claro ejemplo de esta situación fue la contribución al fortalecimiento de la "Asociación cultural de residentes chilenos en Rosario", respecto de la cual, debido a su participación en la jornada organizada por el Foro, sus miembros fundadores tuvieron la oportunidad de dar a conocer las urgencias de la organización en cuanto a la necesidad de una renovación generacional que le diera contenido y fuerza a la comunidad en sus actividades de promoción y financiación, como la participación que todos los años tenían en "Colectividades", un evento cultural organizado en la ciudad de Rosario. Pocos días después, parte del equipo del Foro participó de la Asamblea de la Asociación, donde se eligieron las autoridades y se diagramaron las actividades para el año. Allí, dos miembros chilenos del Foro fueron elegidos como autoridades y una de las integrantes comenzó a formar parte del grupo de baile de la comunidad. A partir de ese momento, los lazos se estrecharon y empezaron a desdibujarse. Las actividades de la Asociación se priorizaron y coincidió con que en ese momento el Foro entró en el cese de actividades.

En esta segunda edición, la organización contraparte estuvo presente pero no salió fortalecida. Una vez terminadas las actividades del Foro, la 13 Ranchos nodo Santa Fe entró en un impasse. El grupo del Foro comenzó a tener una identidad propia y a funcionar como si fuese una organización, pero no pudo dar este importante paso, consolidarse con una personería jurídica que le permitiera una gestión eficiente del proyecto. El mes de septiembre de 2016 es considerado el cierre de la experiencia del Foro, puesto que en ese momento aconteció la última actividad del proyecto. La misma coincidió con una etapa de internacionalización del mismo producto de vínculos interuniversitarios establecidos con la Universidad Federal de Integración Latinoamericana (UNILA) de Foz do Iguazú, Brasil.

El Foro atravesó las fronteras por medio de las redes sociales y desde la universidad vecina se realizó el contacto para conocer sobre la experiencia. La directora del proyecto fue quien concretó el primer viaje a la UNILA, con fines de gestión académica y de extensión, por medio de la Beca AVE Docente (Ayuda para Viajes al Exterior) para docentes e investigadores de la UNR en el mes de marzo de 2015. Seis meses después, la alumna y coordinadora del proyecto realizó un estadía similar con la Beca de AVE Estudiantil (Ayuda para Viajes al Exterior) para auxiliares alumnos institucionalizados por resolución de facultad y al menos 
con un año de antigüedad en tales funciones. ${ }^{15}$ En esa ocasión se llevó adelante un taller para dar a conocer el Foro y se consiguieron voluntades para pensar y desarrollar acciones conjuntas en extensión universitaria. Se creó un espacio virtual para compartir informaciones y establecer un contacto más directo. Un año después, una delegación de tres docentes y un técnico de la UNILA visitaron la UNR en el marco del Congreso Nacional e Internacional de la Democracia, realizado en la Facultad de Ciencia Política y Relaciones Internacionales, en el cual se presentó un panel conjunto sobre las experiencias de intercambio y la realidad de la universidad brasileña. ${ }^{16}$

\section{Un aprendizaje democrático y colectivo}

Para enriquecer el análisis de la experiencia se efectuaron entrevistas a cinco estudiantes avanzados de Relaciones Internacionales de la UNR, miembros del equipo ejecutivo del Foro de Jóvenes. A partir de ellas, podemos afirmar que, como característica innovadora, la experiencia se trató de una propuesta diferente del modelo de enseñanza dominante en las casas de educación superior. La herramienta metodológica utilizada fue la educación popular, que interpeló las relaciones de poder presentes en el acto educativo formal donde existían roles estereotipados de educador y educando y el conocimiento se transfería (Freire, 1970). La experiencia del foro permitió experimentar una nueva pedagogía, donde todos pudieron aprender y enseñar y el conocimiento se construyó dialógicamente; el rol del docente, a veces ocupado por un facilitador, cumplió un papel de orientación permanente que, al no ser unidireccional, permitió la transformación de todos los involucrados.

Todos los entrevistados coincidieron en que el Foro logró establecer una relación entre los docentes y estudiantes diferente de la que habían vivido hasta ese momento en la carrera universitaria. La misma fue caracterizada positivamente como un encuentro más humano que les posibilitaba vincularse como pares:

"El foro me enseñó más que nada la herramienta de la horizontalidad, cómo todos podemos participar y conocer de otros tipos de conocimientos entre todos sin que sea vertical, sin que sea alguien que te esté imponiendo un conocimiento, o un autor". (Estudiante 3, comunicación personal, 20 de septiembre de 2018)

"Lo que más me dejó el foro es aprender del otro. El intercambio de saberes, que uno da y recibe, que no soy ninguna experta." (Estudiante 5, comunicación personal, 18 de septiembre de 2018)

"Uno aprende del intercambio. Empezás a ver las falencias de la carrera, lo que falta reforzar." (Estudiante 4, comunicación personal, 19 de septiembre de 2018)

15) Las funciones eran prestadas en la cátedra Historia de las Relaciones Internacionales Latinoamericanas y Argentina. 16) Un año después, dos miembros del Foro de Jóvenes participaron del $35^{\circ}$ Seminário da Extensão Universitária da Região Sul, en Foz do Iguazú, organizado por la UNILA. En esta oportunidad se establecieron contactos entre las Secretarías de Extensión de ambas universidades. 
"El momento del Foro era un momento de aprendizajes de todos." (Estudiante 2, comunicación personal, 16 de septiembre de 2018)

La práctica extensionista trajo aprendizajes sobre lo que implica la gestión colectiva de un proyecto.

"La carrera da una visión individualista y se centra en la competencia entre los alumnos (...) El foro me dio frente a lo individual la mirada colectiva, saber hablar, saber escuchar, el trabajo en equipo, diferentes formas de trabajar." (Estudiante 1, comunicación personal, 27 de septiembre de 2018)

"Aprender a delegar, ver las cosas como un todo." (Estudiante 5, comunicación personal, 18 de septiembre de 2018)

"Fue una experiencia que se trató de encontrarte y entenderte con tu compañera y compañero, que todos estén los más contentos posibles con ese consenso con esa forma de hacer las cosas." (Estudiante 2, comunicación personal, 16 de septiembre de 2018)

"Se valorizó la idea de bienestar: El aprendizaje es un acto importante (...) si uno no se siente cómodo en el momento que va a aprender o no está contento no incorpora, no procesa la información." (Estudiante 5, comunicación personal, 18 de septiembre de 2018)

Debido a la horizontalidad que se propuso desde un comienzo en la experiencia extensionista, un aspecto positivo reconocido por todos los participantes fue el protagonismo y empoderamiento que comenzaron a tener como estudiantes en los espacios académicos gracias a la posibilidad de hablar en público, coordinar talleres, participar en congresos, relacionarse con autoridades, etcétera.

"Lo fuerte del Foro y lo novedoso es que la iniciativa surgió de los alumnos y no de los docentes, eso hacía que nos escuchen." (Estudiante 3, comunicación personal, 20 de septiembre de 2018)

"Fue una iniciativa de estudiantes, eso es lo que valoraron de todos lados, se arrancó sin la gran ayuda de un docente. Fue una inversión de los roles." (Estudiante 5, comunicación personal, 18 de septiembre de 2018)

El aumento de la confianza también alcanzó otros espacios, como el laboral:

"Las herramientas de educación popular hoy se aplican en los lugares de trabajo." (Estudiante 3, comunicación personal, 20 de septiembre de 2018)

"Las dinámicas del Foro son utilizadas por otras ONG y son valoradas en los lugares del trabajo." (Estudiante 5, comunicación personal, 18 de septiembre de 2018)

"Nos ayudó a pensar las relaciones internacionales como algo transversal, que está presente en todos los ámbitos (...) eso es lo que me dio el foro como herramienta para la vida, te desenvuelve en la sociedad." (Estudiante 1, comunicación personal, 27 de septiembre de 2018) 
El acto educativo monopolizado en el aula (re)produce la colonialidad poder ${ }^{17}$ sobre los seres que trascurren por ella a partir del mecanismo cognitivo de separar y/o partir lo real, dividir los componentes del mundo en unidades aisladas negando sus relaciones, convertir las diferencias en jerarquías, y naturalizar aquellas representaciones (Quintero y Petz, 2009). La colonialidad del ser, o también llamada de las subjetividades, es considerada la dimensión ontológica del poder colonial. En las universidades, este proceso comienza cuando coloca al estudiante en un espacio cerrado sin contacto con el exterior produciendo una división —convencional— entre la universidad y el resto de la sociedad, como si fuera algo ajeno a la misma y donde la primera se coloca en una condición de superioridad respecto de la otra, basada en la idea del conocimiento científicamente válido. Esta relación se repite y multiplica al interior del ámbito académico entre aquellos que están legitimados como autoridad por ser poseedores de un saber -los docentes- y aquellos que no lo tienen -los estudiantes.

A su vez, en cada punta del binomio educador-educando se establecen otras jerarquías que refuerzan la anterior, como, por ejemplo, entre el titular de la cátedra y el jefe de trabajos prácticos dentro del cuerpo docente, o entre el ayudante alumno y los estudiantes del curso en el ámbito del estudiantado. La enumeración de relaciones de poder que reproducen la colonialidad del ser y del saber en la academia sería sumamente extensa si trascendiéramos las fronteras del aula. ${ }^{18}$ Sin embargo, a los fines de este trabajo es preciso centrarnos en la relación que se teje entre el docente y el alumno en el proceso de formación profesional.

Se establece una relación unidireccional representada en la distribución del espacio y en la ubicación de los elementos dentro del aula. Esto puede observarse en la disposición de los bancos separados entre sí y mirando en una misma dirección hacia el pizarrón. Esta distribución del espacio no solo centraliza el acto educativo en el docente sino que individualiza el proceso de aprendizaje. Los alumnos no pueden mirarse entre sí y tampoco entrar en diálogo; el acto educativo se convierte en un consumo de información. Según afirma uno de los estudiantes entrevistados:

"La carrera es muy teórica, muy enciclopedista en muchos aspectos, y uno pierde de vista a veces el impacto que tienen las relaciones internacionales en la vida cotidiana de las personas. El Foro era poner el cuerpo, no dejaba de vincularse a lo académico pero poníamos el cuerpo". (Estudiante 2, comunicación personal, 16 de septiembre de 2018)

17) Aníbal Quijano (2000) propone un esquema de análisis del poder como espacio y red de relaciones sociales de explotación/dominación/conflicto, que son articuladas en la disputa por el control en cinco ámbitos de la existencia social: "(1) el trabajo y sus productos; (2) en dependencia del anterior, la "naturaleza" y sus recursos de producción; (3) el sexo, sus productos y la reproducción de la especie; (4) la subjetividad y sus productos, materiales e intersubjetivos, incluido el conocimiento; (5) la autoridad y sus instrumentos, de coerción en particular, para asegurar la reproducción de ese patrón de relaciones sociales y regular sus cambios" (p. 345). El mecanismo por el cual opera en los diferentes ámbitos de la existencia del sistema mundo moderno colonial es el binomio modernidad/colonialidad, es decir, un conjunto de dos términos que operan de forma dependiente como dos caras de una misma moneda, siendo una visible y la otra oculta o encubierta.

18) Al respecto, el estudio Internacionalización y tensiones para un uso social de la ciencia latinoamericana. Del siglo XIX al XXI de Pablo Kreimer (2013) constata históricamente el vínculo estrecho entre la institucionalización de los campos científicos modernos y las relaciones que se establecían entre los investigadores locales (latinoamericanos) y los líderes de cada disciplina en Europa. Consúltese en: https://www.researchgate.net/publication/277250039_Internacionalizacion_y_tensiones_para_un_uso_social_de_la_ciencia_latinoamericana_Del_siglo_XIX_al_XXI 
En el espacio áulico los cuerpos de los estudiantes son inmovilizados en una silla y desintegrados para enaltecer - en el mejor de los casos— la capacidad de pensar, cuando no la de reproducir el contenido de los libros.

En el próximo apartado identificaremos las posibilidades de repensar y accionar una nueva práctica transformadora a partir de un análisis crítico de esta experiencia.

\section{Reflexiones finales}

En este estudio se incorporó la colonialidad del poder tal como la entiende Quijano, como un patrón de poder global que afecta a todo el sistema mundo moderno y por lo tanto es una forma diferente de entender los asuntos mundiales, desde una lógica de relaciones de poder pero no reducida a las relaciones interestatales. Presenciamos algunos disensos al interior de la corriente de pensamiento decolonial sobre el rol del Estado tal cual hoy lo conocemos. La pregunta que interpela a los autores de esta perspectiva, y sobre la cual no existe un consenso, es si puede existir una propuesta decolonial desde espacios estatales, un modelo político administrativo impuesto a los pueblos de América Latina por la modernidad. La respuesta nos interesa mucho puesto que la universidad pública es el Estado también y, por ende, cabe el cuestionamiento acerca de si puede esta institución generar propuestas decoloniales.

Quienes leímos a Quijano sabemos que él jamás pensó como posible una decolonialidad desde la Modernidad y sus productos. Compartimos la idea del autor y creemos que un nuevo sistema mundo, para ser decolonial, debe dejar de ser moderno. Sin embargo, como nos advierte Wallerstein:

"la verdad, como ideal cultural, ha funcionado como un opio, tal vez el único opio serio del mundo moderno. (...) El opio, sin duda, no es indefectiblemente malo. Calma el dolor. Permite a la gente evadirse de la dura realidad cuando teme que la confrontación con esa realidad solo pueda precipitar las inevitables pérdidas o decadencias". (Wallerstein, 1988:72)

La nostalgia iluminista gana fuerza en los espacios conservadores y se resiste a la transformación.

Reducir en un actor como el Estado o la universidad la diversidad de espacios que hoy penetran las estructuras estatales y universitarias es un colonialismo en el que fallamos sistemáticamente. Por ello, si pensamos en transformaciones, en formas otras de articular las relaciones en el mundo, debemos asumir que solo serán posibles si acontece una deconstrucción. Reconocemos que, en el fondo, aportar a esa profunda y compleja tarea en el campo de las relaciones internacionales es la principal razón que movilizó esta investigación, como lo fue en el equipo del Foro a la hora de proponer una integración regional que otorgara protagonismo a las dimensiones social y cultural y que empoderara a las comunidades como los principales actores del proceso. Se modificaron no solo los contenidos sino también los términos en los que transcurrió el debate sobre la integración regional. El Foro se encargó de visibilizar lo que estaba oculto, ya fuera "por ausencia de diálogo y debate; por prejuicios disciplinares o, porque hay discusiones que no se democratizan y terminan incurriendo en conclusiones sino erróneas, por lo menos incompletas" (Saavedra y Cianciardo, 2016:134). 
En resumen, la incorporación de este corpus teórico decolonial posibilitó hacernos preguntas nuevas en el ámbito específico de la temática investigada, diferentes de las ofrecidas por el mainstream de la disciplina. En la praxis, una ventana de posibilidad para nuevas investigaciones en la carrera de Relaciones Internacionales requiere de lo que Díaz (2010) denomina un "reposicionamiento de las prácticas educativas de carácter emancipatorio".

En este sentido, una práctica transformadora solo podrá surgir de una demanda construida con la comunidad, partiendo de "los niveles de saber y sentir de los sectores populares para alcanzar niveles mayores y críticos de comprensión de la realidad para su transformación" (Medina y Tommasino, 2018:21). Desde las disciplinas sociales modernas coloniales existe "un vínculo de carácter utilitario con la comunidad, en donde se extraen cosas (información, conocimientos) cayendo en un uso y abuso de la misma sin dejar nada a cambio". Por lo tanto, una propuesta en clave decolonial en la carrera de Relaciones Internacionales deberá abandonar

"la idea de que se establece un vínculo asimétrico entre el técnico y la comunidad en donde uno queda en el lugar de dar y el otro en el de recibir, [y] concebir la relación como un intercambio en el que se ponen en juego necesidades y expectativas recíprocas (...). Para los estudiantes, en el contexto de sus prácticas universitarias, el tema de la demanda se les presenta muchas veces como un obstáculo para procesar su inserción en la comunidad. (...) 'No hay demanda' suelen decir”. (Rodríguez et al., 2001:102)

El Foro de Jóvenes por una Latinoamérica integrada surgió de lo que podríamos llamar una demanda interna, esto es, la "necesidad" de los estudiantes avanzados de Relaciones Internacionales de poner en práctica las categorías abordadas durante el proceso de formación y de esta manera aprender en contexto a partir de la acción concreta en la sociedad. Y si bien a lo largo del proyecto se promovió (y muchas veces se logró) una diálogo con la comunidad, los mayores aprendizajes a recuperar son en cuanto a la temática de la construcción de la demanda en la relación técnico-comunidad. Este "técnico", en el caso del Foro, se fue conformando con diferentes disciplinas. Sin embargo, como afirman sus miembros, costó mucho esfuerzo, en ocasiones no tan suficiente, no caer en la aplicación de la receta disciplinar (Saavedra y Cianciardo, 2016).

En este aspecto queda evidenciado cómo la colonialidad operada sobre los estudiantes de relaciones internacionales en su proceso de formación (analizada en el apartado anterior) es, a su vez, reproducida por ellos mismos al vincularse con la sociedad. Desde la academia "se incurre en una miopía disciplinar que no se condice con el contexto de interdependencia compleja que impone actualmente —nos guste o no- la globalización" (Saavedra y Cianciardo:136).

La geopolítica del conocimiento requiere necesariamente la descolonización del aparato conceptual que sostiene la epistemología moderna, así como "las múltiples separaciones de la realidad, que diseñadas por la modernidad guían la producción de las disciplinas de las ciencias sociales" (Galiardi, 2015:43). La realidad es indisciplinada, pregona la extensión crítica. "La interdisciplinariedad no es una receta o algo que se pueda adquirir simplemente por la adhesión a una metodología. Más que eso, es algo que se aprende, practica y experimenta en busca de un diálogo en espacios integrados (en varios sentidos)" (Miranda, 2013:2). En este sentido, decir que la realidad es indisciplinada no es más que aceptar que en la 
sociedad ni los problemas ni las soluciones se encuentran tan prolijamente definidos, sino que se presentan como un todo complejo, interrelacionado. Por esta razón, el estudio de la experiencia extensionista del Foro resultó, a nuestro entender, una propuesta válida para la construcción de conocimiento y la formación profesional precisamente por las instancias de diálogo interdisciplinares e interculturales que desarrolló y que enriquecieron el debate endogámico de la integración regional en las relaciones internacionales.

La universidad y sus miembros, de modo erróneo, asumen en su planificación de prácticas territoriales que el objeto de transformación es la comunidad con la que se trabaja. Así crean proyectos, piensan acciones en solitario y luego se disponen a llevarlas adelante en una realidad concreta que los desborda. El reposicionamiento de prácticas educativas de carácter emancipatorio debe darse al interior de la institución porque, como vimos, es ella la que necesita ser transformada. Por todo ello, la propuesta para una nueva acción territorial deberá contar con una práctica curricularizada en la carrera de Relaciones Internacionales, que trabaje desde el momento de su planificación en un diálogo continuo con las colectividades de inmigrantes latinoamericanos y que tenga como principal instrumento de gestión la sistematización de experiencias.

\section{Referencias bibliográficas}

Baroni, L. et al. (septiembre de 2014). Por Una Latinoamérica Integrada: una apuesta a la construcción de ciudadanía desde la Extensión Universitaria. XI Congreso Nacional y IV Congreso Internacional sobre Democracia. Rosario, Argentina.

Da Silva, C. y Orso, J. (2010). La evolución de la integración latinoamericana. Tres coyunturas históricas: 1810-1910-2010. Historia Regional, (28). Instituto Superior del Profesorado No 3 Eduardo Lafferriere. Villa Constitución, Argentina.

Declaración de Asunción (2003). Foro de Juventudes Políticas del Mercosur. Blog oficial. Recuperado de: http://forodejuventudespoliticasdelmercosur.blogspot.com/

Díaz, C. (2010). Hacia una pedagogía en clave decolonial: entre aperturas, búsquedas y posibilidades. Bogotá: Tábula Rasa.

Dussel, E. (2000). Europa, modernidad y eurocentrismo. En Lander, E. (Ed.). La colonialidad del saber: eurocentrismo y ciencias sociales. Perspectivas latinoamericanas. Buenos Aires: CLACSO.

Freire, P. (1970). Pedagogía del oprimido. Rio de Janeiro: Paz y Tierra.

Galiardi, R. (2015). Una aproximación al Estado del Arte de la Teoría de las Relaciones Internacionales desde una perspectiva latinoamericana en el siglo XXI. Tesina de grado de la Licenciatura en Relaciones Internacionales. Universidad Nacional de Rosario.

Jara, O. (2018). La sistematización de experiencias: práctica y teoría para otros mundos posibles. Bogotá: Centro Internacional de Educación y Desarrollo Humano (CINDE).

Lander, E. (2000). La colonialidad del saber: eurocentrismo y las ciencias sociales. Perspectivas latinoamericanas. Buenos Aires: CLACSO.

Marineau, R. (2010). Los fundamentos de sociodrama. En Moreira, C. (Comp.). Psicodrama en la Universidad. Buenos Aires: Colección Psicodrama en la Universidad, Dra. Mónica Zuretti.

Medina, J. y Tommasino, H. (2018). Extensión crítica: construcción de una Universidad en contexto. Sistematización de experiencias de gestión y territorio de la Universidad Nacional de Rosario. Rosario: UNR Editora. Miranda, M. (2013). Interdisciplinariedad: Ampliando las fronteras del saber. Mimeo. 
Quijano, A. (2000). Colonialidad del poder y clasificación social. Journal of World-System Research, (2), 342386.

Quintero, P. y Petz, I. (2009). Refractando la modernidad desde la colonialidad. Sobre la configuración de un locus epistémico desde la geopolítica del conocimiento y la diferencia colonial. Gazeta de Antropología. Recuperado de: http://digibug.ugr.es/bitstream/handle/10481/6892/G25_52Pablo_Quintero-Ivanna_Petz.pdf? sequence $=10$ \&isAllowed $=y$

Rodríguez, A. et al. (2001). De Ofertas y Demandas: Una propuesta de intervención en psicología comunitaria. Revista de Psicología, X(002). Universidad Nacional de Chile. Santiago: Nuñoa.

Rouquié, A. (2011). América Latina 2010: geopolítica y ambiciones internacionales. En Wollrad, D.; Maihold, G.; Mols, M. (Coords.). La agenda internacional de América Latina: entre nuevas y viejas Alianzas. Buenos Aires: Nueva Sociedad.

Saavedra, O. y Cianciardo, H. (2016). Foro de Jóvenes por una Latinoamérica integrada. En Contino, P.; Daneri, M. (Comps.); Ovando, A. (Coord.). Cartografías del territorio: de la crónica extensionista a la reflexión integral de la universidad (pp. 132-143). $1^{\text {a }}$ ed. Rosario: UNR Editora. Editorial de la Universidad Nacional de Rosario.

Sader, E. (2009). El nuevo topo. Los caminos de la izquierda latinoamericana. Buenos Aires: Siglo Veintiuno Editores y CLACSO.

Tokatlian, J. (2011). Latinoamérica y sus "alianzas" extrarregionales: entre el espejismo, la ilusión y la evidencia. En Dörte, W. et al., La Agenda internacional de América Latina: entre nuevas y viejas Alianzas. Buenos Aires: Nueva Sociedad, Fundación Friedrich Ebert, Stidtung Wissenschaft und Politik.

Wallerstein, I. (1988). El capitalismo histórico. Madrid: Siglo Veintiuno Editores.

Zuretti, M. (2010). Socio-psicodrama. Camino de transformación. En Moreira, C. (Comp.), Psicodrama en la Universidad. Buenos Aires: Colección Psicodrama en la Universidad, Dra. Mónica Zuretti. 